\title{
ReaR
}

\section{Edema agudo de pulmón en paciente con masa auricular izquierda ¿Qué podemos encontrar en la ecografía cardiaca transtorácica?}

González Marín Y (1), Sánchez Espinosa F (2), García Gutiérrez AI (2), Redondo Enríquez JM (1)

(1) Hospital Universitario de Cáceres.

(2) Hospital Universitario de Badajoz.

\section{Resumen}

Los tumores cardiacos primarios son una entidad poco frecuente. Un $75 \%$ de éstos son de estirpe benigna $\mathrm{y}$, entre ellos, el mixoma auricular es el más común, presentándose alrededor del 50\%, con mayor prevalencia en mujeres de 13 a 80 años. Los síntomas del mismo son muy variados, desde pacientes asintomáticos hasta aquellos que han presentado embolismo, síncope, arritmia, insuficiencia cardiaca, astenia o anorexia entre otros.

Presentamos el caso clínico de una paciente que se somete a intervención quirúrgica de mixoma auricular izquierdo y, el manejo anestésico necesario para abordar dicha cirugía.

\section{Introducción}

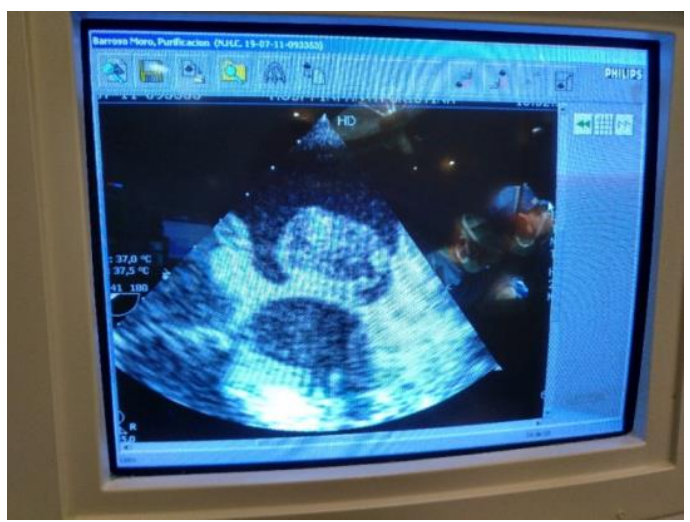

Los tumores cardiacos primarios son una entidad poco frecuente. Un $75 \%$ de éstos son de estirpe benigna $\mathrm{y}$, entre ellos, el mixoma auricular es el más común, presentándose alrededor del $50 \%$, con mayor prevalencia en mujeres de 13 a 80 años. Los síntomas del mismo son muy variados, desde pacientes asintomáticos hasta aquellos que han presentado embolismo, síncope, arritmia, insuficiencia cardiaca, astenia o anorexia entre otros.
Presentamos el caso clínico de una paciente que se somete a intervención quirúrgica de mixoma auricular izquierdo $\mathrm{y}$, el manejo anestésico necesario para abordar dicha cirugía.

\section{Introducción}

Los tumores cardíacos primarios son una entidad poco frecuente. Mucho más habituales son las metástasis de tumores: mama, pulmón, sarcomas de partes blandas, melanoma, leucemias y linfomas. Un $75 \%$ de éstos son de estirpe benigna y, entre ellos, el mixoma auricular es el más común, presentándose alrededor del $50 \%$, con mayor prevalencia en mujeres de 13 a 80 años. Se localizan en la aurícula izquierda en las tres cuartas partes de las ocasiones. Los síntomas del mismo son muy variados, desde pacientes asintomáticos hasta aquellos que han presentado embolismo, síncope, arritmia, insuficiencia cardíaca, astenia o anorexia entre otros (1) (2) 


\section{Descripción y Antecedentes del caso}

Presentamos el caso clínico de una paciente mujer de 51 años con los siguientes antecedentes: sin alergias conocidas, con un peso de $62 \mathrm{~kg}$, talla de $158 \mathrm{~cm}$ e IMC (índice de masa corporal) de $24,8 \mathrm{Kg} / \mathrm{m}^{2}$. Fumadora de 10 cigarrillos/día desde hace 30 años, hipertensa en tratamiento con enalapril de $20 \mathrm{mg}$ y sin ninguna intervención quirúrgica previa.

La paciente es trasladada en situación de edema agudo de pulmón (EAP). Presenta desde hace 48 horas disnea de mínimos esfuerzos (había tenido una transgresión dietética), junto con ortopnea, disnea, tos y algún esputo hemoptoico. Con las siguientes constantes tensión arterial 122/80 $\mathrm{mmHg}$, frecuencia cardíaca de $81 \mathrm{lpm}$ y una saturación de $\mathrm{O} 2$ basal del $98 \%$, es estabilizada en urgencias con $20 \mathrm{mg}$ de furosemida cada $8 \mathrm{~h} y$ con oxigenoterapia mediante gafas nasales a 2 litros. Realizando la anamnesis la paciente refleja episodios de mareo acompañado de palpitaciones, así como síndrome constitucional de 3 meses de evolución.

A continuación, se realiza radiografía de tórax con índice cardiotorácico (ICT) normal, derrame pleural bilateral de pequeña cuantía e imagen de infiltrado intersticial difuso. Analítica anodina con coagulación normal y una hemoglobina de 9,5 g/dl. En segundo lugar, se realiza un angio-TAC de tórax como despistaje de tromboembolismo pulmonar detectándose una masa en aurícula izquierda que protruye hacia ventrículo izquierdo.

En la auscultación cardiaca se descubre soplo diastólico II/VI en foco mitral y, en la pulmonar, murmullo vesicular conservado con ligeros crepitantes en bases. No presenta edemas ni signos de trombosis venosa profunda en miembros inferiores.

Se le realiza electrocardiograma que ilustra ritmo sinusal a 81 latidos por minuto, eje $60^{\circ}$, PR $180 \mathrm{~ms}$, QRS estrechos y sin alteraciones agudas de la repolarización. Tras dicha clínica y resultados de las pruebas complementarias anteriores se decide realizar un ecocardiograma transtorácico donde se visualiza una masa elipsoidal de tamaño (33 x $21 \times 19$ $\mathrm{mm})$ muy móvil en el interior de aurícula izquierda con base de implantación en el septo interauricular; compatible en un primer momento con diagnóstico de mixoma auricular. Se objetiva insuficiencia mitral severa $\mathrm{y}$ una regurgitación grado II dirigida hacia la pared postero-lateral de la aurícula izquierda. El ventrículo izquierdo no se halla dilatado, con válvulas aórtica y tricúspide sin hallazgos de interés. FEVI conservada del $63 \%$

Se realiza coronariografía que descarta lesiones coronarias y se indica tratamiento quirúrgico.

\section{Evolución del caso}

La paciente ingresa a cargo del servicio de cirugía cardiaca hasta el día de la intervención. Se inicia terapia con enoxaparina a dosis anticoagulantes de $40 \mathrm{mg} / \mathrm{sc} / 12 \mathrm{~h}$.

A su llegada a quirófano se encuentra estable con tensión arterial de 123/65 mmHg y en ritmo sinusal a $64 \mathrm{lpm}$. En el antequirófano canalizamos vía periférica de calibre $18 \mathrm{G}$ en miembro superior derecho y administramos 2 miligramos (mg) intravenosos de midazolam para ansiolisis. Una vez dentro de quirófano monitorizamos con electrocardiografía de 5 derivaciones, pulsioximetría, presión arterial no invasiva, TOF y BIS. Canalizamos la vía arterial bajo medidas de bacteriemia 
zero y mediante anestesia tópica. A continuación, procedemos a la inducción administrando $3 \mathrm{mg}$ de midazolam, 150 microgramos $(\mathrm{mcg}) \mathrm{de}$ fentanilo, $15 \mathrm{mg}$ de etomidato y $50 \mathrm{mg}$ de rocuronio. Se realiza la laringoscopia (Cormack-Lehane grado I) y posterior intubación endotraqueal con un tubo de $7 \mathrm{~mm}$ sin incidencias. Se utiliza técnica de anestesia general balanceada mediante sevofluorano con una CAM de 0,6 y perfusión continua de remifentanilo a $0,10-0,15 \mathrm{mcg} / \mathrm{kg} / \mathrm{min}$ y rocuronio a $5 \mathrm{mcg} / \mathrm{kg} / \mathrm{min}$.

Tras la inducción presenta leve hipotensión $(84 / 55 \mathrm{mmHg})$ que es controlada con $10 \mathrm{mg}$ de efedrina. Seguimos con la canalización de catéter swan ganz en vena yugular interna derecha, sin incidencias, con medición de $\mathrm{SvO} 2$ de $83 \%$ e índice cardiaco de 3 $1 / \mathrm{min}$ por $\mathrm{m} 2$.

El ecógrafo transesofágico evidencia la masa auricular en la fosa oval del septo interauricular, sin llegar a protruir en ventrículo. Se encuentra en contacto mantenido con el velo anterior mitral. Figura 1 (eco transesofágica). Vídeos 1 y 2 (eco transesofágica)

Se interviene a la paciente con circulación extracorpórea y canulación de ambas cavas. Se accede a la masa tumoral a través de una atriotomía con abordaje transeptal. Parcial conservación del velo posterior e implantan prótesis mitral mecánica. Figuras 2, 3 y 4 (mixoma auricular).

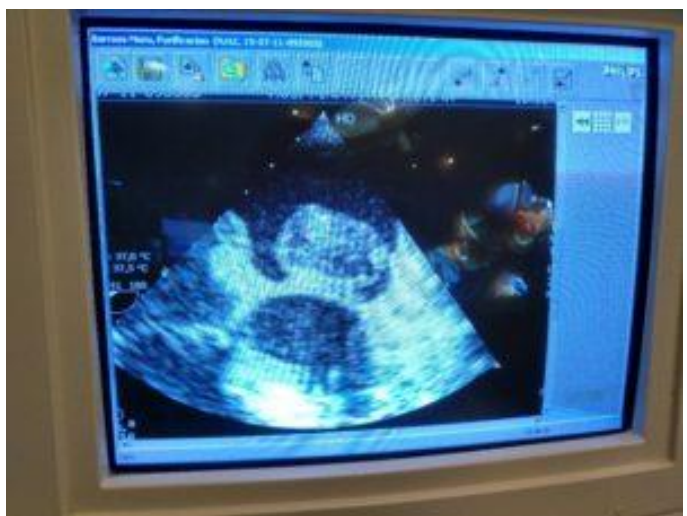

Figura 1. Eco transesofágica del mixoma atrial

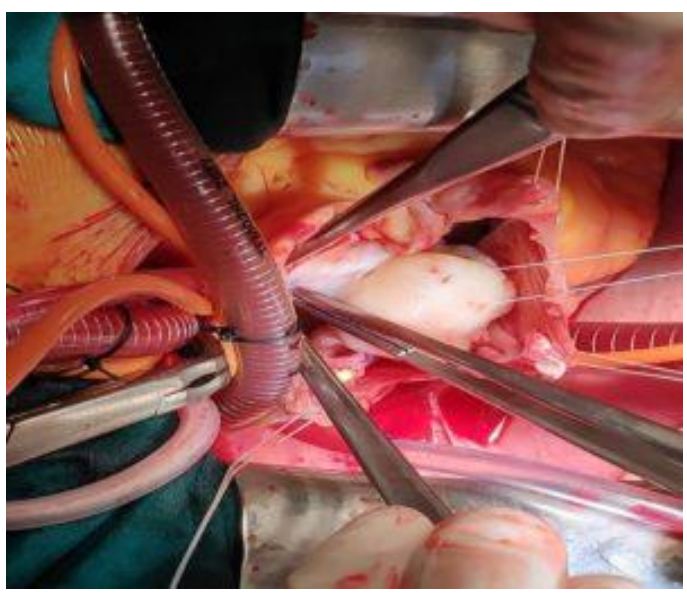

Figura 2. Esternotomía media (resección mixoma auricular)

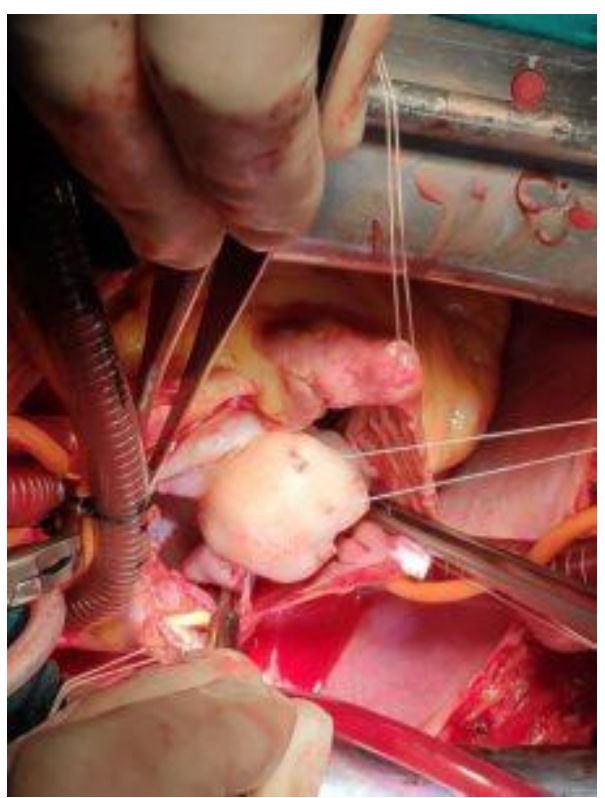

Figura 3. Resección mixoma auricular

La entrada en circulación extracorpórea sucede sin contratiempos. Después de 4 horas de cirugía, 96 minutos de circulación extracorpórea y 84 minutos de tiempo de clampaje, la paciente sale de bomba precisando inicio de 
noradrenalina a $0,20 \mathrm{mcg} / \mathrm{kg} / \mathrm{min} \quad \mathrm{y}$ dobutamina a $5 \mathrm{mcg} / \mathrm{kg} / \mathrm{min}$ por visualizar en la ecografía transesofágica una contractilidad levemente comprometida del ventrículo derecho. La paciente es trasladada a la unidad de recuperación cardíaca intubada, conectada a ventilación mecánica e inestable hemodinámicamente con soporte vasoactivo ( IC de $2,11 / \mathrm{min} / \mathrm{m}^{2}$, PVC de 10 mmHg y ScVO2 62\%). Evoluciona favorablemente con destete respiratorio a las 4 horas postquirúrgicas $\mathrm{y}$ tras la fase de recalentamiento; se transfunde 1 concentrado de hematíes y se administra $10 \mathrm{mg}$ de calcio. A lo largo de los dos días siguientes se consigue descenso de las drogas vasoactivas hasta su retirada, siendo dada de alta a planta con una hemoglobina de $10 \mathrm{~g} / \mathrm{dl}$ y una coagulación en rango. Posteriormente, la paciente inicia tratamiento anticoagulante con acenocumarol.

No se le realizaron estudios analíticos de inmunoglobulinas AAF dirigidas contra los fosfolípidos de carga negativa de las membranas celulares, a pesar de asociarse con algunos tumores cardíacos como el mixoma, puesto que el diagnóstico por imagen y la clínica dieron la clave para intervenir a la paciente de forma urgente.

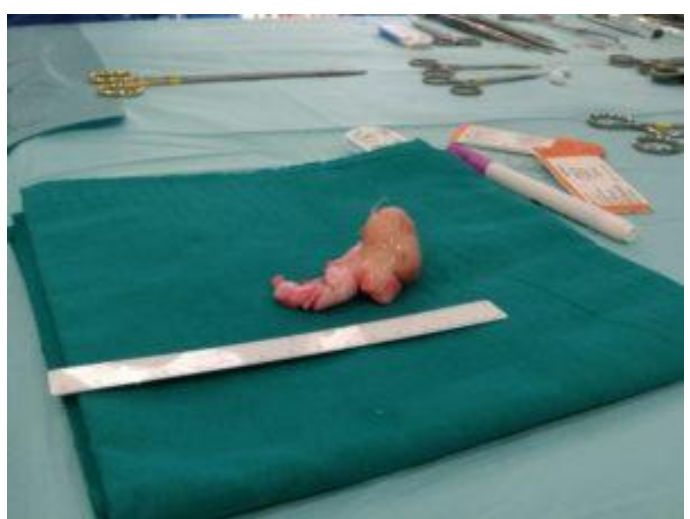

Figura 4 (mixoma extraído)

\section{Discusión}

Los tumores cardíacos primarios son muy poco frecuentes, dentro de ellos el más común es el mixoma. Se trata, por tanto, del tumor benigno intracavitario más frecuente, comprende el $50 \%$ de los tumores cardíacos que ocurren fundamentalmente entre la tercera y la sexta década de la vida. El $75 \%$ de los mixomas se localizan en la aurícula izquierda. Afectan generalmente al sexo femenino y se comportan como una valvulopatía mitral. Generalmente se obtiene la curación mediante la extirpación quirúrgica. Se requiere seguimiento médico periódico por el riesgo de recidiva (4)

El mixoma cardíaco es una neoplasia de histogénesis incierta que ocurre sólo en la superficie endocárdica, con mayor frecuencia de localización auricular. Se ha postulado que las células que dan origen a este tumor son las denominadas «células de reserva subendoteliales», totipotenciales y con capacidad para formar estructuras vasculares y que expresan marcadores endoteliales $\mathrm{y}$ neurales.

Las formas de presentación clínica de los mixomas, son diversas y dependen básicamente del tamaño y de la localización del tumor. Las manifestaciones más usuales son los derivados de los fenómenos embólicos y de la obstrucción al flujo sanguíneo intracardíaco (5)

Los mixomas se caracterizan por un estroma mixoide que le dan una consistencia gelatinosa. Se puede observar, como en nuestro caso, fibrosis y calcificación hecho por el cual se sospechó en un primer momento que podría tratarse de un tumor primario; por lo que se extrajo muestra para biopsia intraoperatoria.

Histológicamente se caracterizó por estar revestido de endotelio y por la presencia de células poligonales inmersas en una matriz de mucopolisacáridos que confirmó 
finalmente el diagnóstico de mixoma auricular (1) (2)

Las células del mixoma cardíaco son histológicas e histogenéticamente diferentes a las células fusiformes de los mixomas de partes blandas.

Las complicaciones más importantes que pueden acontecer en este tipo de tumores son (3):

-Miocardiopatía: Provocando una miocardiopatía hipertrófica con clínica restrictiva.

-Procesos tromboembólicos: Son una de las formas más frecuentes de presentación clínica de los tumores cardíacos.

-Trastornos del ritmo: Más frecuentes en tumores que afectan al sistema de conducción $o$ en pacientes con cavidades dilatadas, generalmente debido a obstrucción mecánica por el propio tumor.

-Síndrome de respuesta inflamatoria sistémica (SIRS): A parte del SIRS propio de una cirugía extracorpórea; en los pacientes con mixomas se ha visto una elevación de los niveles de interleucinas 6 (IL-6) que disminuyen tras la extirpación del tumor. Este aumento de IL favorece la producción de óxido nítrico que se cree que es el mecanismo patogénico que provoca una mayor incidencia del síndrome de respuesta inflamatoria sistémica y gran parte de los síntomas que presentan estos enfermos.

-Lesiones valvulares: La posible implicación valvular en los tumores cardíacos y su posterior reparación es otra de las razones que llevan a la necesidad de uso del eco transesofágico en la extirpación de estos tumores; ya que tras la resección del tumor se han reportado casos que han precisado de recambio valvular por malfuncionamiento de la válvula.

Durante la cirugía es importante limitar la manipulación del tumor por el cirujano dado que puede aumentar las posibilidades de eventos embólicos, pudiendo provocar además un incremento en las posibilidades de diseminación.

Los tumores cardíacos son una patología infrecuente en cirugía cardíaca, siendo el gold estándar el uso de la ecografía transesofágica como monitorización de la hemodinamia del paciente; así como diagnóstico de complicaciones como obstrucción del tracto de salida del ventrículo izquierdo (1)

\section{Bibliografía}

1. Xie X, Bai J, Li X, . Right atrial myxoma. J Card Surg. 2018 Jul;33(7):388-90(PubMed)

2. Navarro O, Fernández J. Estenosis mitral por mixoma auricular. Rev col cardiol. 2016; 23 (4): 304 ( $\underline{\text { HTML }})$

3. Blanco-Coronil A, Moreno Sánchez-Cañete A, González-Román A, Romero-Berrocal A, García-Fernández J. Tumores primarios cardíacos. Complicaciones y manejo anestésico. Rev mex anestesiol. 2015; 38 (4) :289-92

4. Espinosa Martín MC, Quispe Bravo J, Carreno García E. Mixoma auricular. Imagen Diagn. 2016; 7(2):76

5. Gabe E, Rodríguez Correa C, Vigliano C, San Martino J, N Wisner J, González P et al. Mixomas cardíacos: correlación anatomoclínica. Rev esp cardiol. 2002; 55 (5): 505-13 (吕ML) 
Fernando Sánchez Espinosa autor@anestesiar.org

Correspondencia al autor FEA de Anestesiología, Reanimación y Terapéutica del dolor.

Yasmina González Marín

Hospital Universitario de Badajoz.

yasminagm31@gmail.com

MIR de Anestesiología, Reanimación y

Terapéutica del dolor.

Aceptado para el blog en abril de

Hospital Universitario de Cáceres.

2020 